\title{
Frações da matéria orgânica em áreas de Latossolo sob diferentes sistemas de manejo no Cerrado do estado de Goiás
}

\author{
Organic matter fractions in areas Oxisol under different \\ management systems in Cerrado the State of Goiás, Brazil
}

\author{
Roni Fernandes Guareschi ${ }^{1 *}$; Marcos Gervasio Pereira ${ }^{2}$; Adriano Perin ${ }^{3}$
}

Resumo

O objetivo deste trabalho foi avaliar as frações químicas e físicas da matéria orgânica do solo (MOS), bem como realizar a análise espectroscópica na região do ultravioleta-visível do ácido húmico em amostras de um Latossolo Vermelho sob sistema de plantio direto (SPD) com diferentes anos de implantação, e compará-las a áreas de cerrado nativo e pastagem. Foram avaliadas cinco áreas, a saber: cerrado nativo (CE) "stricto sensu"; pastagem plantada (PA) com Brachiaria decumbens; SPD com 3 (SPD3) anos de implantação; SPD com 15 anos (SPD15) de implantação e SPD com mais de 20 (SPD20) anos de implantação. Os teores e estoques de carbono total e das frações húmicas da MOS, aumentaram em função do tempo de implantação do SPD em todas as profundidades analisadas, tendo as frações húmicas apresentado a seguinte ordem: fração ácido fúlvico > fração ácido húmico > fração humina. Os resultados obtidos demonstraram que em função do tempo de implantação do SPD está ocorrendo um aumento das frações mais estáveis das substâncias húmicas e das frações físicas da MOS, proporcionando uma maior estabilidade deste sistema. Verifica-se aumento da relação E4/ E6 dos ácidos húmicos em função do tempo de implantação do SPD, demonstrando um aumento de estruturas alifáticas. A área PA avaliada apresentou os menores teores e estoques das frações húmicas, carbono associado aos minerais (CAM) e relação E4/E6, demonstrando estar em um estádio avançado de degradação em relação às demais áreas avaliadas.

Palavras-chave: Carbono orgânico do solo, fracionamento químico, sistema de plantio direto, pastagem

\begin{abstract}
The objective of this study was to evaluate the physical and chemical fractions of soil organic matter (SOM), as well as perform the spectroscopic analysis in ultraviolet-visible humic acid in a Oxisol under no-tillage system (NTS) with different years of implementation, and compare them to areas of native cerrado and pasture. Was evaluated five areas namely: native cerrado (CE), planted pasture (PA) with Brachiaria decumbens; NTS with 3 (NTS 3) years of implementation; NTS with 15 years (NTS 15) of implementation and NTS with more than 20 (NTS 20) years of implementation. The levels and total carbon stocks and humic fractions of SOM, increased with deployment time the NTS at all depths analyzed, with the humic fractions presented the following order: fraction fulvic acid $>$ fraction humic acid $>$ humin fraction. The results showed that depending on the time of implementation of the NTS
\end{abstract}

\footnotetext{
${ }^{1}$ Tecnólogo em produção de grãos, Doutorando em Ciência do Solo, Dept ${ }^{\circ}$ de Solos, Universidade Federal Rural do Rio de Janeiro, UFRRJ, Seropédica, RJ. E-mail: guareschiecotarelli@hotmail.com

${ }^{2}$ D.Sc., Prof. do Dept ${ }^{\circ}$ de Solos, UFRRJ, Seropédica, RJ. E-mail: gervasio@ufrrj.br

${ }^{3}$ D.Sc., Prof. do Dept ${ }^{\mathrm{o}}$ de Fitotecnia, Instituto Federal Goiano, Campus Rio Verde, IF Goiano, Rio Verde, GO. E-mail: perinrj@ yahoo.com.br

* Autor para correspondência
} 
was observed an increase of more stable fractions of humic substances and physical fractions of SOM, providing greater stability of this system. There is increasing the E4/E6 ratio of humic acids according on the time of implementation of the NTS, demonstrating an increase of aliphatic structures. The area evaluated PA had the lowest concentrations and inventories of humic fractions, carbon associated with minerals (CAM) and E4/E6 ratio, demonstrating to be in an advanced stage of degradation relative to the other areas assessed.

Key words: Organic matter of soil, chemical fractionation, no tillage, pasture

\section{Introdução}

Nas últimas décadas, a paisagem do estado de Goiás foi submetida a significativas alterações, principalmente devido à substituição de vegetação nativa por áreas de agricultura e pecuária. Atualmente, da área original, observam-se apenas $35 \%$, com grande potencial de utilização nos próximos anos, sobretudo para a implantação de projetos agropecuários (FERREIRA; FERREIRA; LOBO, 2007; CLEMENTINO; FERREIRA; LOBO, 2007).

Esta expansão da fronteira agrícola, que ocorre através do desmatamento da vegetação nativa, da adoção de mecanização intensiva e o uso de práticas de manejo inadequadas, alteram os atributos edáficos, os quais levam à degradação, reduzindo a produtividade do solo, principalmente devido à erosão e a perda de nutrientes e matéria orgânica (LAL, 2003; ZINN; DIMAS; SILVA, 2002), o que torna a atividade agropecuária mais onerosa (FARIA et al., 2010; SIQUEIRA NETO et al., 2009). Para minimizar o processo de degradação do solo e os custos de produção, os agricultores da região do cerrado optaram pelo cultivo em sistemas de plantio direto (SPD) (SIQUEIRA NETO et al., 2010).

Segundo Carvalho et al. (2010), a manutenção de resíduos vegetais na superfície, somada à ausência de revolvimento do solo, além de reduzir a emissão de $\mathrm{CO}_{2}$, aumenta o estoque de carbono orgânico total (EstC) e nitrogênio total (EstN) no solo, trazendo ainda benefícios, como: aumento da diversidade microbiana, melhoria da fertilidade e dos atributos físicos do solo (FOLEY et al., 2005; SIX et al., 2002).
As diferentes frações de carbono orgânico do solo apresentam características químicas, físicas e morfológicas distintas, e a distribuição destas pode indicar a qualidade da matéria orgânica (CANELLAS et al., 2003). As substâncias húmicas que representam uma das frações da matéria orgânica do solo (MOS) são consideradas indicativas dos processos e do grau de humificação da MOS, sendo subdivididas nas frações ácidos fúlvicos, ácidos húmicos e humina (NASCIMENTO et al., 2010). Existem trabalhos que demonstram que a fração humina (Hum) é a mais abundante em solos de Cerrado ou em solos manejados, enquanto as frações ácido fúlvico (FAF) e fração ácido húmico (FAH) variam de acordo com o manejo adotado e a profundidade do solo (FONTANA et al., 2006; PASSOS et al., 2007; SOUZA; MELO, 2003).

Já o fracionamento granulométrico da MOS e a identificação dos seus compartimentos pode auxiliar na avaliação das modificações decorrentes do uso devido à maior sensibilidade dessas frações frente ao manejo (CAMBARDELLA; ELLIOTT, 1992; BAYER et al., 2004). A utilização de sistemas de manejo que promovam diferentes aportes de biomassa vegetal pode ser identificada por meio da fração particulada da MOS, sendo possível esta ser utilizada como ferramenta para avaliar a qualidade do solo, principalmente em um curto período de tempo (CONCEIÇÃO et al., 2005; ROSSI et al., 2012). Apesar da importância da determinação desta fração, ainda são poucos os trabalhos com fracionamento físico em solos do Cerrado, desta forma torna-se necessário identificar as suas diferentes frações, bem como suas formas de proteção sob vegetação natural e diferentes sistemas 
de manejo (FIGUEIREDO; RESCK; CARNEIRO, 2010).

Outro método que pode auxiliar nos estudos das substâncias húmicas do solo é a espectroscopia na região do ultravioleta-visível (UV-visível) (KUMADA, 1987). Entre as informações obtidas por esta análise, destaca-se a inferência sobre o grau de humificação. Esta análise consiste na avaliação espectral e massa molar das substâncias húmicas em comprimento de ondas, 465 e $665 \mathrm{~nm}$, obtendose a relação E4/E6 (465 nm / $665 \mathrm{~nm})$. Razões E4/E6 baixo significam constituintes aromáticos mais humificados, enquanto elevados valores dessa relação indicam estruturas alifáticas menos humificadas (PRIMO; MENEZES; SILVA, 2011). Desta forma, tais resultados podem discriminar solos submetidos a diferentes manejos, ou condições naturais, pois segundo Kumada (1987), a absorção de luz aumenta com a elevação no conteúdo de C orgânico do solo.

Diversos trabalhos na literatura tem demonstrado que solos sob vegetação nativa de Cerrado por apresentarem aporte constante de resíduos vegetais e não perturbação do sistema, geralmente apresentam maiores estoques de carbono (EstC) total e das frações químicas e físicas da MOS (SIQUEIRA NETO et al., 2010; FIGUEIREDO; RESCK; CARNEIRO, 2010; SILVA et al., 2011). No entanto, no que se refere as pastagens, o manejo adotado nessas áreas determina a qualidade e quantidade da MOS, ou seja, áreas onde à ausência de manejo e manutenção da produtividade, normalmente apresentam níveis de MOS abaixo dos encontrados originalmente em vegetação nativa (SILVA et al., 2004; SIQUEIRA NETO et al., 2009).

Já em função do tempo de implantação do sistema de plantio direto (SPD) verificam-se modificações nos estoques de carbono (EstC), bem como variações nas frações químicas e fisicas da MOS (FIGUEIREDO; RESCK; CARNEIRO, 2010; SIQUEIRA NETO et al., 2010).

Desta forma, o objetivo deste trabalho foi avaliar a influência de áreas de pastagem, cerrado nativo e SPD com diferentes anos de implantação sobre as frações qualitativas da matéria orgânica do solo (MOS), através do fracionamento químico e físico da MOS e da análise espectroscópica na região do ultravioleta-visível do ácido húmico.

\section{Material e Métodos}

A área de estudo localiza-se na Fazenda Montividiu Tiuba, localizada no município de Montividiu-GO (17 $27^{\circ}$ 52,2" S; 51 ${ }^{\circ} 10^{\prime} 33,1^{\prime \prime}$ W). Como principais características da área em estudo destacam-se a altitude de 890 m e o clima, identificado como clima tropical quente, Aw segundo Köppen, e estação chuvosa e seca bem definida, precipitação com média anual de 1740 $\mathrm{mm}$. O relevo da área é predominantemente plano e os solos das áreas de estudo são classificados como Latossolo Vermelho Distroférrico (EMBRAPA, 2006).

Foram analisadas quatro áreas de cultivo, a saber: 1) pastagem degradada de Brachiaria decumbens (PA) com mais de 20 anos de instalação; 2) SPD com 3 anos de implantação com cultivo de soja no verão e pousio na safrinha (SPD3); 3) SPD com 15 anos de implantação com rotação soja no verão milho/sorgo safrinha (SPD15) e 4) SPD com 20 anos de implantação com rotação soja no verão milho safrinha (SPD20) com cinco repetições para cada área. O delineamento utilizado foi inteiramente casualizado, sendo os cinco sistemas de manejo do solo em uma área de cerrado nativo $(\mathrm{CN})$ "stricto sensu", (localizado dentro da área de reserva da Fazenda Montividiu Tiúba (S 17 27' 52,2"; W0 51 10' 33,1"; altitude $890 \mathrm{~m}$ ), tomada como referência. Todas as áreas encontram-se em torno de $3 \mathrm{~km}$ de distância entre si.

A área de pastagem de Brachiaria decumbens (17 25' 58,5" S; 510 09' 39,4" W; altitude $804 \mathrm{~m}$ ), vem sendo cultivada com uma taxa de lotação aproximada de 1,5 unidade animal por hectare. A

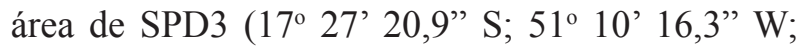


altitude $858 \mathrm{~m}$ ) foi implantado no ano de 1987, com a derrubada do cerrado e cultivo com pastagem durante 20 anos, após este período foi cultivada com a cultura do arroz por 1 ano, e há 3 anos foi adotado o SPD com cultivo de soja. Já a área de SPD15 (17 28' 16,8' S; 51 '11' 20,4" W; altitude $899 \mathrm{~m}$ ), possui um histórico de 27 anos de cultivo convencional com plantio de soja no verão e milho safrinha, sendo o SPD adotado em 1995. O SPD20

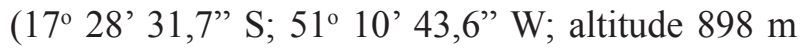
) possui o mesmo histórico que a área de SPD15, porém a adoção do SPD foi realizada em 1990. Atualmente as áreas de SPD15 e SPD20 vêm sendo cultivadas com soja no verão e milho ou sorgo na safrinha (cultivo de janeiro a junho).

A adubação básica das principais culturas dos sistemas agrícolas foram as seguintes: 1) SPD3 e SPD 15 - a) soja (verão) - $458 \mathrm{~kg} \mathrm{ha}^{-1}$ da fórmula 0220-20; b) milho safrinha: $312 \mathrm{~kg} \mathrm{ha}^{-1}$ da formulação 12-15-15, no plantio e $120 \mathrm{~kg} \mathrm{ha}^{-1}$ de uréia, em cobertura aos 25 DAE; 2) SPD 20 - a) soja (verão)
$-200 \mathrm{~kg} \mathrm{ha}^{-1} \mathrm{da}$ fórmula 02-20-20 + $60 \mathrm{~kg} \mathrm{ha}^{-1} \mathrm{de}$ $\mathrm{K}_{2} \mathrm{O}$ e $\mathrm{P}_{2} \mathrm{O}_{5}$ em cobertura; b) milho safrinha: $30 \mathrm{~kg}$ $\mathrm{ha}^{-1}$ de $\mathrm{N}+60 \mathrm{~kg} \mathrm{ha}^{-1}$ de $\mathrm{P}_{2} \mathrm{O}_{5}+70 \mathrm{~kg} \mathrm{ha}^{-1}$ de $\mathrm{K}_{2} \mathrm{O}$ no sulco e $120 \mathrm{~kg} \mathrm{ha}^{-1}$ de uréia, em cobertura aos 25 DAE. O SPD3 recebeu calagem no ano de 2006 e 2010, já o SPD15 recebeu calagem no ano de 2007 e o SPD20 recebeu calagem no ano de 2008. Já a área de PA recebeu uma aplicação de $2 \mathrm{Mg} \mathrm{ha}^{-1}$ de calcário dolomítico no ano 2009.

Em cada uma das áreas, foi delimitada uma área de 150 x $150 \mathrm{~m}$ em posição semelhante na paisagem e, aleatoriamente, foram demarcados cinco locais para a abertura de trincheiras de aproximadamente 1 x $1 \mathrm{~m}$ de superfície e $1 \mathrm{~m}$ de profundidade. Nas profundidades de 0-5, 5-10, 10-20 cm, foram coletadas amostras deformadas, as quais foram secas ao ar, destorroadas e passadas por peneira de $2 \mathrm{~mm}$ de malha. Após esta etapa foram realizadas as análises de granulometria e fertilidade do solo, segundo Embrapa (1997) (Tabela 1).

Tabela 1. Atributos químicos e teores de argila, silte e areia das áreas de Cerrado (CE), pastagem (PA) e sistema de plantio direto com 3 (SPD3), 15 (SPD15) e 20 (SPD20) anos de implantação (Montividiu, GO, 2012).

\begin{tabular}{|c|c|c|c|c|c|c|c|c|c|c|c|c|}
\hline \multirow{2}{*}{ Área } & \multirow{2}{*}{$\mathrm{pH} \mathrm{H} \mathrm{H}_{2} \mathrm{O}$} & \multirow{2}{*}{$\mathrm{P}_{\mathrm{mg} \mathrm{kg}}{ }^{-1}$} & $\mathrm{Ca}$ & $\mathrm{Mg}$ & $\mathrm{K}^{+}$ & $\mathrm{Al}$ & $\mathrm{H}+\mathrm{Al}$ & $\mathrm{T}$ & $\mathrm{V}$ & \multirow{2}{*}{\multicolumn{3}{|c|}{$\begin{array}{l}\text { Argila Silte Areia } \\
\text {--o } \mathrm{g} \mathrm{kg}^{-1}-\end{array}$}} \\
\hline & & & \multicolumn{6}{|c|}{ 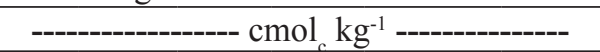 } & $-\% 0^{-}$ & & & \\
\hline & \multicolumn{12}{|c|}{$0-5 \mathrm{~cm}$} \\
\hline $\mathrm{CE}$ & 4,4 & 9,5 & 0,8 & 1,9 & 0,2 & 0,5 & 13,3 & 16,2 & 17,8 & 410 & 170 & 420 \\
\hline PA & 5,7 & 2,1 & 3,9 & 4,7 & 0,2 & 0,0 & 7,3 & 16,3 & 54,6 & 600 & 190 & 210 \\
\hline SPD3 & 5,2 & 26,7 & 1,6 & 2,0 & 0,3 & 0,1 & 3,6 & 7,7 & 52,9 & 590 & 270 & 140 \\
\hline SPD15 & 5,0 & 33,6 & 2,6 & 2,9 & 0,3 & 0,1 & 4,6 & 10,6 & 56,4 & 630 & 170 & 200 \\
\hline \multirow[t]{2}{*}{ SPD20 } & 6,2 & 32,1 & 2,6 & 2,8 & 0,3 & 0,0 & 2,8 & 8,6 & 67,1 & 410 & 120 & 470 \\
\hline & \multicolumn{12}{|c|}{$5-10 \mathrm{~cm}$} \\
\hline $\mathrm{CE}$ & 3,6 & 5,8 & 0,0 & 1,1 & 0,1 & 0,9 & 9,9 & 11,0 & 10,7 & 390 & 200 & 410 \\
\hline PA & 5,6 & 1,5 & 3,0 & 3,0 & 0,1 & 0,0 & 5,0 & 11,2 & 54,8 & 570 & 240 & 190 \\
\hline SPD 3 & 5,1 & 20,9 & 0,9 & 1,7 & 0,3 & 0,1 & 3,8 & 6,6 & 42,0 & 610 & 270 & 120 \\
\hline SPD 15 & 4,9 & 29,1 & 1,9 & 1,9 & 0,3 & 0,2 & 4,0 & 8,2 & 52,6 & 670 & 160 & 170 \\
\hline \multirow[t]{2}{*}{ SPD 20} & 5,3 & 29,2 & 1,8 & 1,8 & 0,3 & 0,1 & 3,9 & 7,9 & 50,3 & 460 & 110 & 430 \\
\hline & \multicolumn{12}{|c|}{$10-20 \mathrm{~cm}$} \\
\hline $\mathrm{CE}$ & 3,8 & 4,8 & 0,0 & 0,9 & 0,1 & 0,8 & 10,1 & 11,1 & 9,1 & 380 & 170 & 450 \\
\hline PA & 5,5 & 1,7 & 2,5 & 1,7 & 0,1 & 0,0 & 7,7 & 12,0 & 35,5 & 590 & 220 & 190 \\
\hline SPD 3 & 4,3 & 18,4 & 0,1 & 0,8 & 0,2 & 0,1 & 2,2 & 3,4 & 34,9 & 610 & 280 & 110 \\
\hline SPD 15 & 4,5 & 28,7 & 1,4 & 1,3 & 0,3 & 0,3 & 3,3 & 6,3 & 48,3 & 660 & 180 & 160 \\
\hline SPD 20 & 4,4 & 28,4 & 1,3 & 1,3 & 0,3 & 0,2 & 3,4 & 6,4 & 47,1 & 470 & 100 & 430 \\
\hline
\end{tabular}

Fonte: Elaboração dos autores. 
A densidade do solo (Ds) foi determinada através do método do anel volumétrico (EMBRAPA, 1997), onde em cada uma das trincheiras, nos diferentes sistemas de manejo, foi realizada a coleta de amostras indeformadas com auxílio de um anel volumétrico nas profundidades de 0-5, 5-10, 10-20 $\mathrm{cm}$.

O carbono orgânico total (COT) foi determinado através de combustão seca por analisador CHNS (Elementar Analysensysteme GmbH, Hanau, Alemanha).

Para o fracionamento químico da MOS foi utilizada a técnica de solubilidade diferencial (DABIN, 1976), segundo modificação de Benites, Madari e Machado (2003). As frações da matéria orgânica foram identificadas, segundo os termos estabelecidos pela Sociedade Internacional de Substâncias Húmicas, em fração ácido fúlvico (FAF), fração ácido húmico (FAH) e fração humina (Hum) (SWIFT, 2001).

Foi realizado o fracionamento granulométrico da MOS (CAMBARDELLA; ELLIOTT, 1993), obtendo-se o carbono orgânico particulado (COP) e carbono orgânico associado aos minerais (CAM). Para isso, foi utilizado $20 \mathrm{~g}$ de solo e $60 \mathrm{~mL}$ de solução de hexametafosfato de sódio $\left(5 \mathrm{~g} \mathrm{~L}^{-1}\right)$, os quais foram agitados durante 15 horas em agitador horizontal. A seguir, a suspensão foi passada por peneira de 53 $\mu \mathrm{m}$. O material retido na peneira (COP) foi seco em estufa a $50^{\circ} \mathrm{C}$, quantificado em relação a sua massa, moído em gral de porcelana e analisado em relação ao teor de $\mathrm{C}$ por combustão seca por um analisador $\mathrm{C} / \mathrm{H} / \mathrm{N} / \mathrm{S}$ (Elementar Analysensysteme $\mathrm{GmbH}$, Hanau, Alemanha). O CAM foi obtido a partir da diferença ente $\mathrm{C}$ total e COP. Também foi calculado o estoque de carbono particulado (EstCOP) e do carbono associado aos minerais (EstCAM) pelo método de massa equivalente (SISTI et al., 2004).

Para o COT e as diferentes frações da matéria orgânica, obtidas pelos fracionamentos químico e granulométrico, foi calculado o estoques de carbono pelo método da massa equivalente (SISTI et al., 2004). Esse método leva em consideração a espessura da camada e a DS, bem como, utiliza como referência, a massa de solo de uma área, estabelecidas como referência, a qual é tomada como base para o cálculo do estoque em todas as demais. Neste estudo, considerou-se como referência a área de Cerrado, a qual representa a condição original do solo.

A análise espectroscópica na região do ultravioleta-visível do ácido húmico foi realizada com auxílio de um espectrofotômetro digital modelo $22 \mathrm{pc}$, da marca Spectrumlab, na faixa do visível para determinação das absorbâncias em 380, 465 e 665 nm. Para determinação da relação $E_{4} / E_{6}$, foi dividida a absorbância em $465 \mathrm{~nm}$ pela obtida em 665 nm. (KUMADA, 1987).

Para todos os dados em cada profundidade, foi feita a avaliação da normalidade dos dados (Kolmogorov-Smirnov - Ks), homogeneidade das variâncias dos erros pelo Teste de Cochran (Coch). Posteriormente, os resultados foram submetidos à análise de variância com aplicação do teste $\mathrm{F}$ e os valores médios comparados entre si pelo teste de Tukey a 5\%, com auxílio do programa ASSISTAT (SILVA; AZEVEDO, 2002).

\section{Resultados e Discussão}

Pela análise da tabela 2 pode-se verificar que as áreas de PA e SPD3 apresentaram os menores valores de estoque de carbono (EstC) em relação aos demais sistemas de cultivo em todas as profundidades. Observa-se também que nas áreas de SPD15 e SPD20 nas camadas superficiais os valores de EstC não diferiram entre si da área de CE, porém nas camada 10-20 observa-se que as áreas mais antigas de SPD apresentam valores de EstC inferiores ao CE. 
Tabela 2. Estoque de carbono (EstC) das áreas avaliadas (Montividiu, GO, 2012).

\begin{tabular}{|c|c|c|c|c|c|c|}
\hline \multirow{3}{*}{ Camada $(\mathrm{cm})$} & \multicolumn{6}{|c|}{ Sistemas avaliados } \\
\hline & $\mathrm{CE}$ & PA & SPD3 & SPD 15 & SPD20 & $\mathrm{CV}(\%)$ \\
\hline & \multicolumn{6}{|c|}{$\operatorname{EstC}\left(\mathrm{Mg} \mathrm{ha}^{-1}\right)$} \\
\hline $0,0-5,0$ & $13,54 \mathrm{a}$ & $7,96 \mathrm{~b}$ & $7,31 \mathrm{~b}$ & $13,61 \mathrm{a}$ & $15,56 \mathrm{a}$ & 7,12 \\
\hline $5,0-10,0$ & $17,86 \mathrm{a}$ & $7,49 \mathrm{~b}$ & $7,86 \mathrm{~b}$ & $13,99 \mathrm{a}$ & $14,12 \mathrm{a}$ & 15,60 \\
\hline $10,0-20,0$ & $36,34 \mathrm{a}$ & $14,41 \mathrm{c}$ & $14,79 \mathrm{c}$ & $29,32 \mathrm{~b}$ & $28,83 \mathrm{~b}$ & 3,81 \\
\hline
\end{tabular}

*Médias seguidas de mesma letra minúscula na linha não diferem significativamente entre os diferentes sistemas de uso do solo pelo teste Tukey a $5 \%$.

Fonte: Elaboração dos autores.

Uma possível explicação para os baixos valores de EstC na área de SPD3 em relação as demais, pode ser atribuída ao pequeno tempo de adoção do sistema. Segundo Carvalho et al. (2010) o aumento dos teores de MOS em áreas de SPD, em comparação a outros sistemas de manejo, pode estar relacionado principalmente ao seu tempo de instalação, pois o acúmulo de carbono neste sistema ocorre muito lentamente, levando de 10 a 15 anos para se tornar expressivo. Já os baixos teores de EstC da área PA podem estar relacionado a sua baixa produção de resíduos vegetais, a ausência de manejo e pastoreio intensivo, ao pequeno aporte de resíduos vegetais e também redução na distribuição do sistema radicular. Silva et al. (2004) também verificaram que pastagens de baixa produtividade em diversas regiões do Cerrado favoreceram a redução do conteúdo de carbono no solo.

As áreas de 15 e 20 anos de SPD recebem um aporte constante de resíduos vegetais de diferentes qualidades, o que pode ter sido um fator preponderante para os valores de EstC serem similares aos observados na área de CE. Resultados semelhantes foram relatados por Siqueira Neto et al. (2010), que ao avaliarem no Cerrado áreas com diferentes anos de implantação do SPD em um Latossolo, constataram que áreas com 12 anos de cultivo apresentaram valores de EstC semelhantes ao Cerrado. Tais autores atribuíram este resultado a grande utilização de fertilizantes e pesticidas nas culturas de soja no verão e milho safrinha, que favorecem maiores produções de grãos e biomassa, aumentando a MOS em função do tempo de implantação do SPD.

$\mathrm{Na}$ profundidade de $10-20 \mathrm{~cm}$ a área de $\mathrm{CE}$ apresentou os maiores valores de EstC em relação aos sistemas de cultivo, enquanto as áreas de SPD15 e SPD20 foram estatisticamente semelhantes e superiores as áreas de SPD3 e PA. De acordo com Siqueira Neto et al. (2009) o cerradão apresenta maiores quantidades de carbono e nitrogênio no solo decorrentes do aporte constante de resíduos vegetais e não perturbação do sistema. Em relação às áreas de SPD, observa-se que existe um aumento no estoque de C em função do tempo de implantação, porém este aumento é menos expressivo que o observado nas camadas superficiais do solo (0-5 e 5-10 cm). Segundo Bayer e Mielniczuk (1999), o acúmulo de MOS no SPD ocorre lentamente, sendo assim, pode-se inferir pelos resultados deste trabalho, que 15 e 20 anos de implantação do SPD ainda não foram capazes de acumular EstC nas camadas subsuperficiais quando comparadas a área de CE.

Dentre as frações da MOS, a humina foi a que se apresentou em maiores teores e estoques em relação às demais, independente da área estudada e profundidades analisadas (Tabela 3). Em diversos estudos em solos tropicais também se observou predomínio do carbono da fração humina em relação às outras frações (ASSIS et al., 2006; ROSSI et al., 2012). Os elevados valores de carbono da fração humina (C-HU) podem estar relacionados ao tamanho das moléculas e ao maior grau de 
estabilidade desta fração (FONTANA et al., 2006). Segundo Fontana et al. (2006) as FAF e FAH, por apresentarem menor estabilidade, podendo ser translocadas para camadas mais profundas, serem polimerizadas ou mineralizadas, e diminuir, assim, seu teor residual no solo.

Tabela 3. Carbono da fração humina (Hum), ácido fúlvico (FAF) e ácido húmico (FAH) e relação ácido húmico e ácido fúlvico (C-FAH/C-FAF) nos diferentes sistemas de uso do solo (Montividiu, GO, 2012).

\begin{tabular}{|c|c|c|c|c|}
\hline \multirow{3}{*}{ Sistemas avaliados } & \multicolumn{3}{|c|}{ Carbono das frações húmicas $\left(\mathrm{g} \mathrm{kg}^{-1}\right)$} & \multirow{2}{*}{$\begin{array}{c}\text { C-FAH/ C-FAF (g } \\
\left.\mathrm{kg}^{-1}\right)\end{array}$} \\
\hline & Hum & FAF & FAH & \\
\hline & \multicolumn{3}{|c|}{$0,0-5,0 \mathrm{~cm}$} & $0,0-5,0 \mathrm{~cm}$ \\
\hline $\mathrm{CE}$ & $17,10 \mathrm{a}^{*}$ & $5,20 \mathrm{a}$ & $5,36 \mathrm{~b}$ & $1,03 \mathrm{c}$ \\
\hline PA & $9,75 \mathrm{c}$ & $2,84 \mathrm{~d}$ & $4,72 \mathrm{~d}$ & $1,66 \mathrm{a}$ \\
\hline SPD3 & $9,87 \mathrm{c}$ & $2,85 \mathrm{~d}$ & $4,71 \mathrm{~d}$ & $1,65 \mathrm{a}$ \\
\hline SPD15 & $16,11 \mathrm{~b}$ & $3,25 \mathrm{c}$ & $4,90 \mathrm{c}$ & $1,51 \mathrm{ab}$ \\
\hline \multirow[t]{2}{*}{ SPD20 } & $16,28 \mathrm{~b}$ & $3,89 \mathrm{~b}$ & $5,81 \mathrm{a}$ & $1,49 \mathrm{~b}$ \\
\hline & \multicolumn{3}{|c|}{$5,0-10,0 \mathrm{~cm}$} & $5,0-10,0 \mathrm{~cm}$ \\
\hline $\mathrm{CE}$ & $13,57 \mathrm{a}$ & $3,80 \mathrm{a}$ & $5,63 \mathrm{~b}$ & $1,47 \mathrm{a}$ \\
\hline PA & $6,02 \mathrm{c}$ & $2,94 \mathrm{c}$ & $4,50 \mathrm{~d}$ & $1,53 \mathrm{a}$ \\
\hline SPD3 & $6,09 \mathrm{c}$ & $2,96 \mathrm{c}$ & $4,50 \mathrm{~d}$ & $1,51 \mathrm{a}$ \\
\hline SPD15 & $11,03 \mathrm{~b}$ & $3,19 \mathrm{~b}$ & $4,86 \mathrm{c}$ & $1,52 \mathrm{a}$ \\
\hline \multirow[t]{2}{*}{ SPD20 } & $11,26 \mathrm{~b}$ & $3,76 \mathrm{a}$ & $5,77 \mathrm{a}$ & $1,53 \mathrm{a}$ \\
\hline & \multicolumn{2}{|c|}{$10,0-20,0 \mathrm{~cm}$} & & $10,0-20,0 \mathrm{~cm}$ \\
\hline $\mathrm{CE}$ & $11,00 \mathrm{a}$ & $3,53 \mathrm{ab}$ & $5,82 \mathrm{a}$ & $1,64 \mathrm{a}$ \\
\hline $\mathrm{PA}$ & $4,92 \mathrm{c}$ & $2,95 \mathrm{c}$ & $4,52 \mathrm{c}$ & $1,53 \mathrm{a}$ \\
\hline SPD3 & $4,96 \mathrm{c}$ & $2,98 \mathrm{c}$ & $4,54 \mathrm{c}$ & $1,53 \mathrm{a}$ \\
\hline SPD15 & $10,83 \mathrm{~b}$ & $3,41 \mathrm{~b}$ & $5,17 \mathrm{~b}$ & $1,51 \mathrm{a}$ \\
\hline SPD20 & $10,38 \mathrm{~b}$ & $3,63 \mathrm{a}$ & $5,86 \mathrm{a}$ & $1,61 \mathrm{a}$ \\
\hline
\end{tabular}

*Médias seguidas de mesma letra minúscula na coluna não diferem significativamente entre os diferentes sistemas de uso do solo pelo teste Tukey a $5 \%$.

Fonte: Elaboração dos autores.

Os menores teores de $\mathrm{C}$ das substâncias húmicas foram observados para as áreas de PA e SPD3 (Tabela 3). Esses resultados podem estar relacionados aos históricos de uso de cada área, pois ambas as áreas (PA e SPD3) foram utilizadas como pastagem por 20 anos o que conseqüentemente pode ter contribuído para a redução dos teores de carbono das frações húmicas. Essa redução ocorre porque os resíduos vegetais provenientes das pastagens são pobres em lignina, precursor químico da humina, ácidos húmicos e outros compostos recalcitrantes (SANTOS; CAMARGO, 1999). Assim, solos sob pastagem, possuem matéria orgânica menos recalcitrante e, portanto, são mais sensíveis às variações climáticas, químicas e microbiológicas que resultam em maior velocidade de degradação da matéria orgânica do solo (MARTINS; CORINGA; WEBER, 2009).

Através da Tabela 3 observa-se para todas as profundidades analisadas que a área de CE apresentou os maiores teores de $\mathrm{C}$ da fração humina (C-HUM), e que as áreas SPD15 e SPD20 apresentaram C-HUM semelhantes entre si e superiores as áreas SPD3 e PA (Tabela 3). Todavia, quando se considera o estoque de carbono da fração humina, observa-se que este padrão é alterado, ou seja, as áreas SPD15 e SPD20 possuem estoques semelhantes ao $\mathrm{CE}$ e superiores às demais áreas (Figura 1). Estes resultados demonstram com o decorrer do tempo de adoção do SPD pode-se 
aumentar os valores do estoque de carbono da fração humina, sendo que os maiores valores de carbono na fração C-HUM, na maioria das vezes promovem maior expressão das propriedades da fração coloidal da matéria orgânica, tais como: retenção de água, melhoria na agregação do solo e maior retenção de cátions, características de grande importância quando se trata do cultivo de sistemas sustentáveis de produção (SOUZA; MELO, 2003).

Figura 1. Estoque de carbono da fração humina (Hum), ácido fúlvico (FAF) e ácido húmico (FAH) em diferentes profundidades do perfil do solo dos sistemas de manejo (Montividiu, GO, 2012).

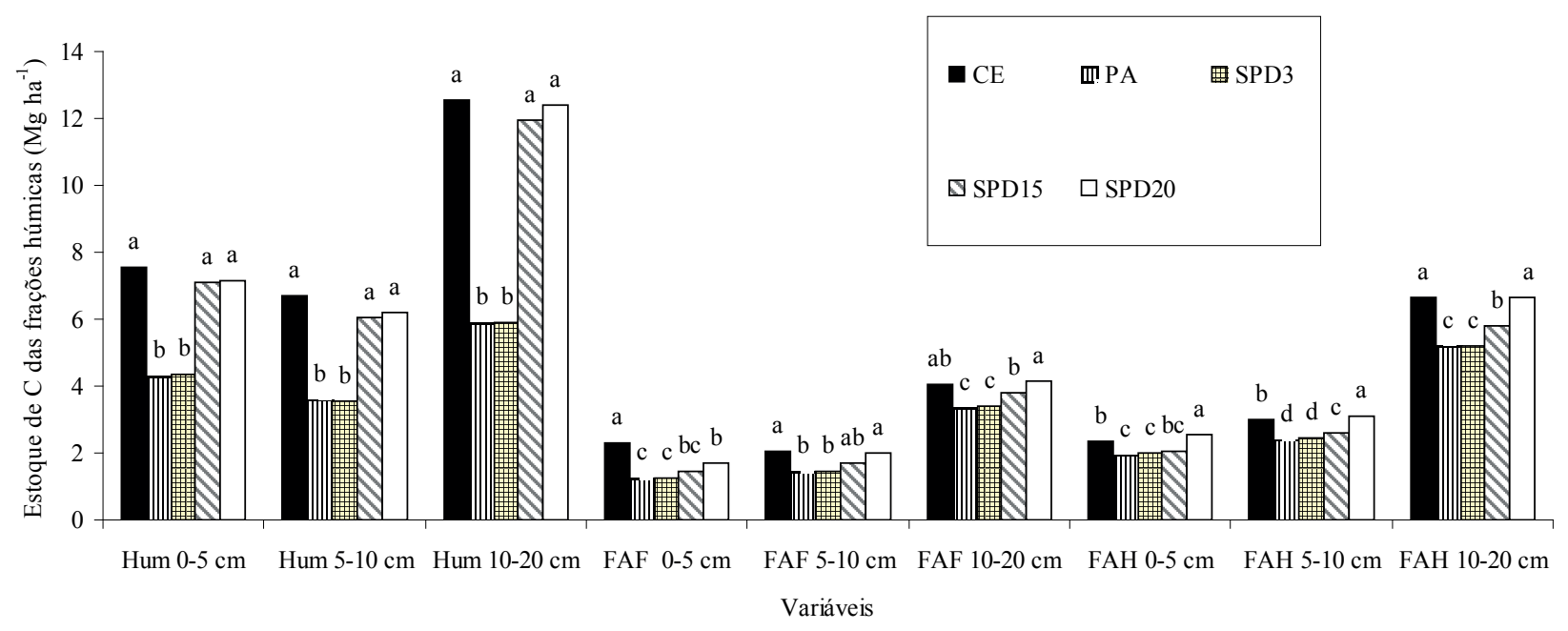

Fonte: Elaboração dos autores.

Já em relação aos teores e estoques de carbono da fração ácido fúlvico (C-FAF), constata-se que está ocorrendo um aumento desta fração em função do tempo de implantação do SPD, sendo que a área de SPD20 nas profundidades de 5-10 e 10-20 cm apresenta quantidades semelhantes desta fração em relação à área de $\mathrm{CE}$ (Tabela 3 e Figura 1). Com o mesmo padrão observado para o C-FAF, os teores e estoques de carbono da fração ácido húmico (C-FAH) estão aumentando em função do tempo de implantação do SPD, porém, verifica-se que a área de SPD20 apresentou teores e estoques de C-FAH superiores (0-5 e 5-10 cm), mais semelhantes (10$20 \mathrm{~cm}$ ) à área de CE (Tabela 3 e Figura 1).

De maneira geral, pode-se observar entre ás áreas de SPD, que está ocorrendo um aumento das frações estáveis da MOS em função do tempo de adoção do SPD, e que a distribuição do carbono das frações húmicas está seguindo o sentido das frações mais lábeis (ácido fúlvico) para as mais estáveis (ácido húmico e humina). A partir do padrão observado, pode-se inferir que o não revolvimento do solo, bem como o constante aporte de resíduos vegetais de diferentes relações $\mathrm{C} / \mathrm{N}$ na superfície do solo, está favorecendo a humificação da MOS em função do tempo de implantação do SPD. De acordo com Loss et al. (2010) áreas em SPD, pelo menor revolvimento do solo e, consequentemente, preservação dos resíduos vegetais em sua superfície, promovem condições que favorecem o aumento dos teores e estoques de C-FAH em detrimento ao C-FAF. Outra justificativa seria uma conversão rápida de ácidos fúlvicos em ácidos húmicos neste sistema. 
A relação C-FAH/C-FAF só apresentou diferença estatística entre as áreas de estudo na profundidade de 0-5 cm, sendo observado o menor valor dessa relação na área de $\mathrm{CE}$, constatando-se diminuição do valor desta relação em função do tempo de implantação do SPD (Tabela 3). A relação C-FAH/ C-FAF foi usada por Kononova (1982) como um indicador da qualidade do húmus, pois expressa o grau de evolução do processo de humificação da matéria orgânica. Em solos tropicais, normalmente, esta razão é inferior a 1 devido à menor intensidade do processo de humificação, condensação e síntese, atribuídas à intensa mineralização dos resíduos, restrições edáficas e ao baixo conteúdo de bases trocáveis à atividade biológica nos solos mais intemperizados (CANELLAS et al., 2002). A área de $\mathrm{CE}$ apresentou relação FAH/FAF na camada superficial do solo próxima de 1,03, o que segundo alguns autores (EBELING et al., 2004; GIÁCOMO; PEREIRA; BALIEIRO, 2008) caracteriza material de qualidade ótima, que permitiria o estabelecimento de propriedades físicas e químicas favoráveis ao desenvolvimento de plantas.

Já em relação às áreas de SPD, observa-se uma tendência de redução da relação FAH/FAF em função do tempo de implantação, isso indica que, nestas áreas, há predomínio de ácidos húmicos em relação aos ácidos fúlvicos, apresentando um material orgânico mais estável. A manutenção de maiores teores de C-FAH em detrimento aos teores de C-FAF evidencia solos mais preservados, de manejo mais conservacionista (CANELLAS et al., 2003).

Quanto ao fracionamento granulométrico, não foi observada diferença estatística entre as áreas avaliadas para o carbono orgânico particulado (COP) na profundidade de $10-20 \mathrm{~cm}$ e para o estoque de carbono orgânico particulado (EstCOP) na profundidade de 5-10 e 10-20 cm. Porém, nas demais profundidades, o COP $(0-5$ e $5-10 \mathrm{~cm})$ e o EstCOP (0-5 cm) está ocorrendo uma redução do carbono particulado em função do tempo de implantação das áreas de SPD, sendo que as áreas SPD15 e SPD20 apresentam valores de COP inferiores à área de $\mathrm{CE}$ (Tabela 4). Ao contrário do observado para o COP e EstCOP, o carbono associado aos minerais (CAM) e estoque de carbono associado aos minerais (EstCAM) estão aumentando em função do tempo de implantação do SPD nas profundidades analisadas (Tabela 4). 
Tabela 4. Teores e estoques de $\mathrm{C}$ das frações granulométricas da MOS nos diferentes sistemas de uso do solo (Montividiu, GO, 2012).

\begin{tabular}{|c|c|c|c|c|}
\hline \multirow{3}{*}{$\begin{array}{l}\text { Sistemas } \\
\text { avaliados }\end{array}$} & \multicolumn{2}{|c|}{ Frações granulométricas de carbono $\left(\mathrm{g} \mathrm{kg}^{-1}\right)$} & \multicolumn{2}{|c|}{ Estoque de $\mathrm{C}$ das frações granulométricas $\left(\mathrm{Mg} \mathrm{ha}^{-1}\right)$} \\
\hline & $\mathrm{COP}$ & CAM & EstCOP & EstCAM \\
\hline & \multicolumn{2}{|c|}{$0,0-5,0 \mathrm{~cm}$} & \multicolumn{2}{|c|}{$0,0-5,0 \mathrm{~cm}$} \\
\hline $\mathrm{CE}$ & $8,28 \mathrm{a}$ & $22,48 \mathrm{~b}$ & $3,59 \mathrm{a}$ & $9,94 \mathrm{~b}$ \\
\hline PA & $5,40 \mathrm{ab}$ & $10,05 \mathrm{c}$ & $3,56 \mathrm{ab}$ & $4,40 \mathrm{c}$ \\
\hline SPD3 & $5,55 \mathrm{ab}$ & $10,42 \mathrm{c}$ & $2,72 \mathrm{ab}$ & $4,59 \mathrm{c}$ \\
\hline SPD15 & $4,21 \mathrm{~b}$ & $25,44 \mathrm{~b}$ & $2,38 \mathrm{~b}$ & $11,23 \mathrm{ab}$ \\
\hline \multirow[t]{2}{*}{ SPD20 } & $4,27 \mathrm{~b}$ & 29,69 a & $2,41 \mathrm{~b}$ & 13,09 a \\
\hline & \multicolumn{2}{|c|}{$5,0-10,0 \mathrm{~cm}$} & \multicolumn{2}{|c|}{$5,0-10,0 \mathrm{~cm}$} \\
\hline $\mathrm{CE}$ & $5,20 \mathrm{a}$ & $30,86 \mathrm{a}$ & $2,58 \mathrm{a}$ & $15,28 \mathrm{a}$ \\
\hline PA & $4,23 \mathrm{ab}$ & $9,85 \mathrm{c}$ & $2,55 \mathrm{a}$ & $4,94 \mathrm{~b}$ \\
\hline SPD3 & $4,42 \mathrm{ab}$ & $11,01 \mathrm{c}$ & $2,49 \mathrm{a}$ & $5,37 \mathrm{~b}$ \\
\hline SPD15 & $4,11 \mathrm{~b}$ & $23,00 \mathrm{~b}$ & $2,28 \mathrm{a}$ & $11,71 \mathrm{a}$ \\
\hline \multirow[t]{2}{*}{ SPD20 } & $4,19 \mathrm{~b}$ & $22,53 \mathrm{~b}$ & $2,31 \mathrm{a}$ & $11,81 \mathrm{a}$ \\
\hline & \multicolumn{2}{|c|}{$10,0-20,0 \mathrm{~cm}$} & \multicolumn{2}{|c|}{$10,0-20,0 \mathrm{~cm}$} \\
\hline $\mathrm{CE}$ & $3,77 \mathrm{a}$ & $28,11 \mathrm{a}$ & $4,30 \mathrm{a}$ & $32,04 \mathrm{a}$ \\
\hline PA & $3,43 \mathrm{a}$ & $8,60 \mathrm{c}$ & $4,27 \mathrm{a}$ & $10,14 \mathrm{c}$ \\
\hline SPD3 & $3,72 \mathrm{a}$ & $8,78 \mathrm{c}$ & $4,23 \mathrm{a}$ & $10,56 \mathrm{c}$ \\
\hline SPD15 & $3,61 \mathrm{a}$ & $21,79 \mathrm{~b}$ & $4,13 \mathrm{a}$ & $25,19 \mathrm{~b}$ \\
\hline SPD20 & $3,38 \mathrm{a}$ & $21,41 \mathrm{~b}$ & $4,24 \mathrm{a}$ & $24,56 \mathrm{~b}$ \\
\hline
\end{tabular}

*Médias seguidas de mesma letra minúscula na coluna não diferem significativamente entre os diferentes sistemas de uso do solo pelo teste Tukey a $5 \%$.

Fonte: Elaboração dos autores.

Segundo Figueiredo, Resck e Carneiro (2010), na dinâmica das frações lábeis e estáveis da MOS sob sistemas de manejo e cerrado nativo, há uma correlação negativa entre COP e CAM, indicando que os processos de formação destas frações ocorre de maneira inversa. Ou seja, para que se tenham maiores teores de CAM no solo é necessário que ocorra maior decomposição (diminuição) dos teores de COP para posterior associação com os minerais do solo nos tamanho silte e argila. Neste estudo este padrão foi observado, e verificou-se que em função do tempo de implantação do SPD os teores e estoques de COP tendem a diminuir paralelo ao aumento dos teores e estoques de CAM. De maneira geral, pode-se constatar que resultados obtidos para os teores e estoques de CAM e para as frações húmicas (C-FAH, C-FAF e C-HUM) da MOS são concordantes, demonstrando que em função do tempo de implantação do SPD está ocorrendo um aumento das frações mais estáveis das substâncias húmicas e das frações físicas da MOS (CAM).

Observa-se que está ocorrendo um aumento da relação E4/E6 em função do tempo de implantação do SPD, onde as áreas SPD15 e SPD20 apresentaram relações E4/E6 semelhantes entre si e superiores às demais áreas analisadas (Tabela 5). Diante dos resultados da relação E4/E6 dos ácidos húmicos, pode-se inferir que está havendo um aumento de cadeias alifáticas em função do tempo de implantação do SPD. Resultados semelhantes foram relatados por Rosa et al. (2008) onde ao avaliar diferente sistemas de manejo, os autores constataram que o SPD apresentou valores entre 4,7 e 5,9, demonstrando possuir C-FAH com estruturas menos condensadas e/ou de estruturas micelares menores. Sendo assim, pode-se observar também que os ácidos húmicos provenientes da área de $\mathrm{CE}$ e PA apresentam maior quantidade de compostos aromáticos que as áreas de SPD. 
Tabela 5. Relação E4/E6 de ácidos húmicos da camada de $0-5 \mathrm{~cm}$ do solo nos diferentes sistemas de manejo (Montividiu, GO, 2012).

\begin{tabular}{cc}
\hline Sistemas avaliados & Relação E4/E6 \\
\hline CE & $3,10 \mathrm{~d}^{*}$ \\
PA & $4,08 \mathrm{c}$ \\
SPD3 & $4,47 \mathrm{~b}$ \\
SPD15 & $5,10 \mathrm{a}$ \\
SPD20 & $5,18 \mathrm{a}$ \\
\hline
\end{tabular}

* Médias seguidas de mesma letra na coluna não diferem entre as áreas avaliadas pelo teste Tukey a 5\%.

Fonte: Elaboração dos autores.

De acordo com Ceretta et al. (2008) em sistemas de culturas com menor aporte de $\mathrm{C}$, onde ao longo do tempo a decomposição da matéria orgânica é maior que a adição de carbono, são mantidas no solo, predominantemente, formas mais humificadas da matéria orgânica, portanto, o valor da relação E4/ E6 é menor. Esta consideração explica perfeitamente o comportamento dos dados obtidos neste trabalho, pois observa-se que a relação E4/E6 e teor de C aumentam em função do tempo de implantação do SPD e que a área de PA por estar em estádio avançado de degradação apresenta menores teores de $\mathrm{C}$ e relação E4/E6 dos ácidos húmicos.

\section{Conclusões}

$\mathrm{A}$ área PA avaliada apresentou os menores teores e estoques das frações húmicas, CAM e relação E4/ E6, demonstrando estar em um estádio avançado de degradação em relação às demais áreas avaliadas;

Os teores e estoques de carbono total e das frações húmicas da MOS aumentaram em função do tempo de implantação do SPD em todas as profundidades analisadas, na seguinte ordem: FAF $>$ FAH > FHUM;

O teores e estoques de COP estão reduzindo, enquanto há o aumento de CAM em função do tempo de adoção do SPD;

Verifica-se aumento da relação E4/E6 dos ácidos húmicos em função do tempo de implantação do
SPD, demonstrando um aumento de estruturas alifáticas. Já as áreas de CE e PA apresentam menores valores de relação E4/E6 em comparação as áreas de SPD, o que indica maior grau de condensação de constituintes aromáticos.

\section{Referências}

ASSIS, C. P.; JUCKSCH, I.; MENDONÇA, E. S.; NEVES, J. C. L. Carbono e nitrogênio em agregados de Latossolo submetido a diferentes sistemas de uso e manejo. Pesquisa Agropecuária Brasileira, Brasília, v. 41, n. 10, p. 1541-1550, 2006.

BAYER, C.; MARTIN-NETO, L.; MIELNICZUK, J.; AURÉLIO PAVINATO, A. Armazenamento de carbono em frações lábeis da matéria orgânica de um Latossolo Vermelho sob plantio direto. Pesquisa Agropecuária Brasileira, Brasília, v. 39, n. 7, p. 677-683, 2004.

BAYER, C.; MEILNICZUK, J. Dinâmica e função da matéria orgânica. In: SANTOS, G. A.; CAMARGO, F. A. O. (Ed.). Fundamentos da matéria orgânica do solo, ecossistemas tropicais e subtropicais. Porto Alegre: Genesis, 1999. p. 1-26.

BENITES, V. M.; MADARI, B.; MACHADO, P. L. O. A. Extração e fracionamento quantitativo de substância húmicas do solo: um procedimento simplificado de baixo custo. Rio de Janeiro: Embrapa Solos, 2003. 7 p. (Comunicado técnico, 16).

CAMBARDELlA, C. A.; ELLIOTT, E. T. Methods for physical separation and characterization of soil organic matter fractions. Geoderma, Madison, v. 56, n. 1, p. 449457, 1993.

Particulate soil organic matter changes across a grassland cultivation sequence. Soil Science Society of America Journal, Madison, v. 56, n. 1, p. 777-783, 1992.

CANEllas, L. P.; A. C. X.; Velloso, A. C. X.; MARCIANO, C. R.; RAMALHO, J. F. G. P.; RUMJANEK, V. M.; REZENDE, C. E.; SANTOS, G. A. Propriedades químicas de um Cambissolo cultivado com cana-de-açúcar, com preservação do palhiço e adição de vinhaça por longo tempo. Revista Brasileira de Ciência do Solo, Viçosa, v. 27, n. 5, p. 935-944, 2003.

CANELLAS, L. P.; VELLOSO, A. C. X.; RUMJANEK, V. M.; GURIDI, F.; OLIVARES, F. L.; SANTOS, G. A.; BRAZ FILHO, R. Distribuition of the humified fractions and characteristics of the humic acids of an ultisol under cultivation of Eucalyptus and sugar cane. Terra, Chapingo, v. 20, n. 4, p. 371-381, 2002. 
CARVALHO, J. L. N.; AVANZI, J. C.; SILVA, M. L. N.; MELLO, C. R.; CERRI, C. E. P. Potencial de sequestro de carbono em diferentes biomas do brasil. Revista Brasileira de Ciência do Solo, Viçosa, v. 34, n. 2, p. 277 289, 2010.

CERETTA, C. A.; BAYER, C.; DICK, D. P.; MARTINNETO, L.; COLNAGO, L. A. Métodos espectroscópicos. In: SANTOS, G. A.; SILVA, L. S.; PASQUALOTO, C. L.; CAMARGO, F. A. O. Fundamentos da matéria orgânica do solo: ecossistemas tropicais e subtropicais. 2. ed. Porto Alegre: Metropole, 2008. p. 201-227.

CLEMENTINO, N. C.; FERREIRA, M. E.; LOBO, F. C. Riscos de desmatamentos e potencial de regeneração da vegetação nativa: definindo prioridades e estratégias territoriais. Boletim Goiano de Geografia, Goiânia, v. 27, n. 1, p. 83-96, 2007.

CONCEIÇÃO, P. C.; AMADO, T. J. C.; MIELNICZUK, J.; SPAGNOLLO, E. Qualidade do solo em sistemas de manejo avaliada pela dinâmica da matéria orgânica e atributos relacionados. Revista Brasileira de Ciência do Solo, Viçosa, v. 29, n. 5, p. 777-788, 2005.

DABIN, B. Méthode d'extraction et de fractionnement des matiéres organiques dans les sols tropicaux. Chah Orston, ser Pédology, Bondy, v. 4, n. 4, p. 287-297, 1976.

EBELING, A. G.; ESPINDULA JUNIOR, A.; VALLADARES, G. S.; ANJOS, L. H. C.; PEREIRA, M. G. Propriedades químicas como indicadores ambientais em organossolos do Estado do Rio de Janeiro. Revista Universidade Rural: Série Ciências da Vida, Seropédica, v. 24, n. 1, p. 1-6, 2004.

EMPRESA BRASILEIRA DE PESQUISA AGROPECUÁRIA - EMBRAPA/CNPS. Manual de métodos de análise de solos. Rio de Janeiro: EMBRAPA Solos, 1997. 212 p.

Sistema brasileiro de classificação de solos. 2 . ed. Rio de Janeiro: EMBRAPA Solos, 2006. 306 p.

FARIA, A. F. G.; SANTOS, A. C.; SANTOS, T. M.; BATISTELLA FILHO, F. Influência do manejo do solo nas propriedades químicas e físicas em topossequência na bacia do rio Araguaia, Estado do Tocantins. Revista Brasileira de ciência do solo, Viçosa, v. 34, n. 2, p. 517524, 2010.

FERREIRA, N. C.; FERREIRA, M. E.; LOBO, F. C. Riscos de desmatamentos e potencial de regeneração da vegetação nativa: definindo prioridades e estratégias territoriais. Boletim Goiano de Geografia, Goiânia, v. 27, n. 1, p. 83-96, 2007.

FIGUEIREDO, C. C. de; RESCK, D. V. S.; CARNEIRO, M. A. C. Labile and stable fractions of soil organic matter under management systems and native Cerrado. Revista
Brasileira de Ciência do Solo, Viçosa, v. 34, n. 3, p. $907-$ 916, 2010.

FOLEY, J. A.; DEFRIES, R.; ASNER, G. P.; BARFORD, C.; BONAN, G.; CARPENTER, S. R.; CHAPIN, F. S.; COE, M. T.; DAILY, G. C.; GIBBS, H. K.; HELKOWSKI, J. H.; HOLLOWAY, T.; HOWARD, E. A.; KUCHARIK, C. J.; MONFREDA, C.; PATZ, J. A.; PRENTICE, I. C.; RAMANKUTTY, N.; SNYDER, P. K. Global consequences of land use. Science, New York, v. 309, n. 5734, p. 570-574, 2005.

FONTANA, A.; PEREIRA, M. G.; LOSS, A.; CUNHA, T. J. F.; SALTON, J. C. Atributos de fertilidade e frações húmicas de um Latossolo Vermelho no Cerrado. Pesquisa Agropecuária Brasileira, Brasília, v. 41, n. 5, p. 847-853, 2006.

GIÁCOMO, R. G.; PEREIRA, M. G.; BALIEIRO, F. C. Estoques de carbono e nitrogênio e distribuição das frações húmicas no solo sob diferentes coberturas florestais. Revista Brasileira de Ciências Agrárias, Recife, v. 3, n. 1, p. 42-48, 2008.

KONONOVA, M. M. Materia orgánica del suelo: su naturaleza, propiedades y métodos de investigación. Barcelona: Oikos-tau, 1982. 364 p.

KUMADA, K. Chemistry of soil organic matter. 2. ed. Tokyo: Japan Scientific Societies Press, 1987. 241 p.

LAL, R. Global potential of carbon sequestration to mitigate the greenhouse effect. Critical Review in Plant Science, v. 22, n. 2, p. 151-184, 2003.

LOSS, A.; MORAES, A. G. L.; PEREIRA, M. G.; SILVA, E. M. R.; ANJOS, L. H. C. Carbono, matéria orgânica leve e frações oxidáveis do carbono orgânico sob diferentes sistemas de produção orgânica. Comunicata Scientiae, Bom Jesus, v. 1, n. 1, p. 57-64, 2010.

MARTINS, E. L.; CORINGA, J. E. S.; WEBER, O. L. S. Carbono orgânico nas frações granulométricas e substâncias húmicas de um Latossolo Vermelho Amarelo distrófico - LVAd sob diferentes agrossistemas. Acta amazônica, Manaus, v. 39, n. 3, p. 655-660, 2009.

NASCIMENTO, P. C.; LANI, J. L.; MENDONÇA, E. S.; ZOFFOLI, H. J. O.; PEIXOTO, H. T. M. Teores e características da matéria orgânica de solos hidromórficos do Espírito Santo. Revista Brasileira de Ciência do Solo, Viçosa, v. 34, n. 2, p. 339-348, 2010.

PASSOS, R. R.; RUIZ, H. A.; CANTARUTTI, R. B.; MENDONÇA, E. S. Carbono orgânico e Nitrogênio em agregados de um Latossolo Vermelho distrófico sob duas coberturas vegetais. Revista Brasileira de Ciência do Solo, Viçosa, v. 31, n. 5, p. 1109-1118, 2007.

PRIMO, D. C.; MENEZES, R. S. C.; SILVA, T. O. 
Substâncias húmicas da matéria orgânica do solo: uma revisão de técnicas analíticas e estudos no nordeste brasileiro. Scientia Plena, São Cristóvão, v. 7, n. 5, p. $1-13,2011$.

ROSA, C. M.; VARGAS, C. R. M.; PINHEIRO, D. D.; PAULETTO, E. A.; SILVA, G. A. Teor e qualidade de substâncias húmicas de planossolo sob diferentes sistemas de cultivo. Ciência Rural, Santa Maria, v. 38, n. 6, p. 1589-1595, 2008.

ROSSI, C. Q.; PEREIRA; M. G.; GIÁCOMO, S. G.; BETTA, M.; POLIDORO, J. C. Frações lábeis da matéria orgânica em sistema de cultivo com palha de braquiária e sorgo. Revista Ciência Agronômica, Fortaleza, v. 43, n. 1, p. 38-46, 2012.

SANTOS, G. A.; CAMARGO, F. A. O. Fundamentos da matéria orgânica do solo: ecossistemas tropicais e subtropicais. Porto Alegre: Ed. Genesis, 1999. 508 p.

SILVA, E. F.; LOURENTE, E. P. R.; MARCHETTI, M. E.; MERCANTE, F. M.; FERREIRA, A. K. T.; FUJII, G. C. Frações lábeis e recalcitrantes da matéria orgânica em solos sob integração lavoura pecuária. Pesquisa Agropecuária Brasileira, Brasília, v. 46, n. 10, p. 13211331, 2011.

SILVA, F. A. S. de; AZEVEDO, C. A. V. de. Versão do programa computacional Assistat para o sistema operacional Windows. Revista Brasileira de Produtos Agroindustriais, Campo Grande, v. 4, n. 1, p. 71-78, 2002.

SILVA, J. E.; RESCK, D. V. S.; CORAZZA, E. J.; VIVALDI, L. Carbon storage in clayey oxisol cultivated pastures in the "Cerrado" region, Brazil. Agriculture, Ecosystem and Environment, Amsterdam, v. 103, n. 2, p. 357-363, 2004.
SIQUEIRA NETO, M.; PICCOLO, M. C.; SCOPEL, E.; COSTA JUNIOR, C.; CERRI, C. C.; BERNOUX, M. Carbono total e atributos químicos com diferentes usos do solo no Cerrado. Acta Scientiarum Agronomy, Maringá, v. 31, n. 4, p. 709-717, 2009.

SIQUEIRA NETO, M.; SCOPEL, E.; CORBEELS, M.; CARDOSO, A. N.; DOUZET, J. M.; FELLER, C.; PICCOLO, M. C.; CERRI, C. C.; BERNOUX, M. Soil carbon stocks under no-tillagemulch-based cropping systems in the Brazilian Cerrado: An on-farm synchronic assessment. Soil \& Tillage Research, Amsterdam, v. 110, n. 5, p. 187-195, 2010.

SISTI, C. P. J.; SANTOS, H. P.; KOHHANN, R.; ALVES, B. J. R.; URQUIAGA, S.; BODDEY, R. M. Change in carbon and nitrogen stocks in soil under 13 years of conventional or zero tillage in southern Brazil. Soil and Tillage Research, Amsterdam, v. 76, n. 2, p. 39-58, 2004.

SIX, J.; FELLER, C.; DENEF, K.; OGLE, S. M.; SA, J. C. M.; ALBRECHT, A. Soil organic matter, biota and aggregation in temperate and tropical soils - effects of notillage. Agronomie, Paris, v. 22, n. 2, p. 755-775, 2002.

SOUZA, W. J. O.; MELO, W. J. Matéria orgânica em um Latossolo submetido a diferentes sistemas de produção de milho. Revista Brasileira de Ciência do Solo, Viçosa, v. 27, n. 6, p. 1113-1122, 2003.

SWIFT, R. S. Sequestration of carbon by soil. Soil Science, Baltimore, v. 166, n. 11, p. 858-871, 2001.

ZINN, Y. L.; DIMAS, V. S.; SILVA, J. E. Soil organic carbon as affected by afforestation with Eucalyptus and Pinus in the Cerrado region of Brazil. Forest Ecology and Management, Amsterdam, v. 166, n. 3, p. 285-294, 2002 . 
